\title{
3-WAY GATED RECURRENT UNIT NETWORK ARCHITECTURE FOR STOCK PRICE PREDICTION
}

\author{
Arjun Singh Saud \\ Assistant Professor, Tribhuvan University, \\ Central Department of Computer Science and IT, Kathmandu, Nepal \\ Email: arjunsaud@cdcsit.edu.np \\ Subarna Shakya \\ Professor, Tribhuvan University, Institute of Engineering, Lalitpur, Nepal \\ Email: drss@ioe.edu.np
}

\begin{abstract}
Stock price prediction has been the aim of stock investors since the beginning, which is important for the investors to make rational decisions about buying and selling stocks. Nowadays deep learning techniques and technical indicators are popular tools among researchers for predicting stock prices. Mainly researchers from the field of computer science, Statistics, and finance are actively involving in this research field. This research paper proposed a 3-way gated recurrent unit (3-GRU) architecture to forecast the next day's close price. The model is a combination of component GRU networks, where each component GRU network predicts the next day's close price using a different set of technical indicators. The proposed 3-GRU model was evaluated by comparing its performance with all component GRU networks. Its performance was also compared with the GRU network that combines different sets of the technical indicators into one feature vector uses it to predict the next day's close price. From the experimental results, we observed that the 3-GRU network architecture is able to predict the next day's close price with lower mean squared error and greater consistency than other models and hence concluded that it is the better approach for predicting next day's stock price.
\end{abstract}

Keywords: 3-Way Gated Recurrent Unit (3-GRU); Stock Price Prediction; Mean Average Convergence Divergence (MACD); Average Directional Index (ADX); Know Sure Things (KST).

\section{Introduction}

Stock price prediction is the act of determining the future trend of stock prices. It has been the aim of investors since the beginning as accurate forecasting of stock prices helps investors to make rational decisions about buying and selling stocks and reducing risks [1]. Two widely used approaches used by financial analysts to predict stock price movements are fundamental analysis and technical analysis [2]. The fundamental analysis combines economic analysis, industry analysis, and company analysis to determine the fair value of the stock price. On the other hand, technical analysis is the act of predicting the future direction of stock prices from the analysis of historical market data. Depending on the requirement, technical analysts may take different time frames such as daily, weekly, monthly etc. In addition, technical analysts use a variety of technical indicators [3, 4].

It is very difficult for financial analysts to analyze the vast amount of noisy and non-stationary stock data and identify useful patterns from it [5]. Therefore, machine learning models became popular to predict future stock prices [6-8]. Traditional machine learning models are not capable of remembering the historical context but recurrent neural networks (RNNs) have this ability. Therefore, RNNs gained popularity over traditional machine learning models to predict stock prices [9]. Since RNNs suffer from vanishing/exploding gradient descent problem, LSTM and GRU networks became state-of-art models for stock price prediction [10-13].

There are many research works that used technical indicators for predicting stock trends [11, 14-23]. The selection of input features was varied greatly among the researches carried out to predict the stock market [24]. The number of input features used in the researches was ranged from one [10] to more than fifty [25]. On the other side, the type of input features used by the researchers was also largely diverse. Some researchers only used historical trading data like Open, High, Low, Close, and Volume whereas other researchers used the mix of historical trading data and technical indicators as input to the prediction model. Further, some researchers used historical trading data with fundamental analysis indicators and statistical data [24]. 
Technical indicators are preferred tools for financial analysts to predict future movements of stocks. Primarily technical indicators are calculated from stock prices and volume of past trading data $[2-4,25]$. Broadly technical indicators can be divided into five categories [26]: Trend Indicators, Momentum Indicators, Volume Indicators, Volatility Indicators, and Other Indicators. The major trend indicators used by technical analysts are average directional index (ADX), mean average convergence divergence (MACD), mass index (MI), moving Averages (MA), parabolic SAR, Trix, vortex indicator (VI), and know sure thing (KST) Oscillator. And the major momentum indicators are momentum, RSI, stochastic oscillator, and Williams \%R [27-29].

Many researchers combine historical trading data and various technical indicators into one feature vector and use it as input to the stock price prediction model [30]. However, technical analysts use different technical indicators separately, combine the results of these analyses, and draw a final conclusion. Based on this observation, this research paper proposed a 3-Way Gated Recurrent Unit (3-GRU) network to estimate the next day's close price. The proposed model is a combination of three GRU networks that predicts next day's close price using mean average convergence divergence (MACD), know Sure things (KST), and average directional index (ADX) technical indicators respectively. The detail of the proposed model is provided in the materials and methods section under the proposed model title. Hereafter, in this research paper, these component GRU networks are referred as GRU-MACD, GRU-KST, and GRU-ADX. The main objectives of this research work are threefold: (1) to analyze the performance of GRU-MACD, GRU-KST, and GRU-ADX in predicting next day's close price (2) to analyze the performance of combining all three technical indicators into one feature vector and using it as input to the GRU network (hereafter this model is referred as GRU-ALL model) for predicting next day's close price (3) to compare the performance of the proposed 3-GRU model with all component GRU networks and GRU-ALL model.

\section{Background}

This section briefly describes theories and models based on which 3-way gated recurrent unit (3-GRU) architecture was formulated.

\subsection{Mean Average Convergence Divergence (MACD)}

MACD is a widely used trend indicator among technical analysts. It is calculated using historical closing prices of stocks [29]. The MACD indicator is a combination of three time-series data: MACD series, MACD Signal, and MACD histogram. It is calculated using exponential moving averages (EMA's) of different periods as given in Equation 1.

$$
\begin{aligned}
& \text { MACD }=12 \text { day EMA(Close })-26 \text { day EMA(Close) } \\
& \text { MACD Signal }=9 \text { day EMA(MACD) } \\
& \text { MACD Histogram = MACD }- \text { MACD Signal }
\end{aligned}
$$

\subsection{Average Directional Index (ADX)}

Average Directional Index (ADX) is also a trend and momentum indicator that signifies the strength of a stock price trend [28]. The indicator does not determine the direction of trend rather it only determines the strength of the current trend. ADX is derived using the two directional movement indicators: Positive directional indicator $(+\mathrm{DI})$ and negative directional indicator (-DI). Positive directional indicator increases when movement is upward and negative directional indicator increases when movement is downward. ADX indicator is calculated using Equation 2.

$$
\begin{aligned}
& \mathrm{Up}=\text { CurrentHigh }- \text { PreHigh } \quad \text { Down }=\text { PrevLow }- \text { CurrentLow } \\
& \{\text { if } \mathrm{Up}>\text { Down and } \mathrm{Up}>0 \text { then }+\mathrm{DI}=\mathrm{Up} \quad \text { else }+\mathrm{DI}=0 \\
& \text { if Down }>\text { Up and Down }>0 \text { then }-\mathrm{DI}=\text { Down } \quad \text { else }-\mathrm{DI}=0 \\
& \mathrm{TR}=\max \{\text { High }- \text { Low, abs(High }- \text { Current }), \operatorname{abs}(\text { Low }- \text { Current })\} \\
& \operatorname{ATR}=\frac{1}{n} \sum_{\mathrm{i}=1}^{\mathrm{n}} \mathrm{TR}_{\mathrm{i}} \quad \text { where } \mathrm{n}=14 \\
& \pm \mathrm{SDI}=\sum_{\mathrm{i}=1}^{\mathrm{n}} \mathrm{DI}-\frac{1}{\mathrm{n}} \sum_{\mathrm{i}=1}^{\mathrm{n}} \mathrm{DM}+\mathrm{CDI} \quad+\mathrm{DI}=\frac{+\mathrm{SDI}}{\mathrm{ATR}} \times 100 \text { and }-\mathrm{DI}=\frac{-\mathrm{SDI}}{\mathrm{ATR}} \times 100 \\
& \mathrm{ADX}=\frac{14 \mathrm{Day} \operatorname{EMA}(\mathrm{abs}((+\mathrm{DI})-(-\mathrm{DI}))}{(+\mathrm{DI})+(-\mathrm{DI})} \times 100
\end{aligned}
$$




\subsection{Know Sure Thing (KST) Oscillator}

Know Sure Thing (KST) is also a type of trend and momentum oscillator. It is based on the Rate of Change (ROC) indicators. ROC is the momentum indicator that measures the change in percentage between the current stock price and the price of the N-period ago. KST oscillator combines ROC of four different periods and combines them into a single momentum indicator [27]. It is useful for identifying major stock market cycles. This means it is useful for determining primary swings of the stock market cycle [31]. KST indicator is calculated using Equation 3.

$$
\begin{aligned}
& \text { ROC1 }=\frac{\text { Close }_{t}-\text { Close }_{t-10}}{\text { Close }_{\mathrm{t}-10}} \times 100 \\
& \text { ROC2 }=\frac{\text { Close }_{t}-\text { Close }_{t-15}}{\text { Close }_{\mathrm{t}-15}} \times 100 \\
& \text { ROC3 }=\frac{\text { Close }_{t}-\text { Close }_{t-20}}{\text { Close }_{t-20}} \times 100 \\
& \text { ROC4 }=\frac{\text { Close }_{\mathrm{t}}-\text { Close }_{\mathrm{t}-30}}{\text { Close }_{\mathrm{t}-30}} \times 100 \\
& \mathrm{KST}=\mathrm{SMA} 10(\mathrm{ROC} 1) * 1+\mathrm{SMA} 10(\mathrm{ROC} 2) * 2+ \\
& \text { SMA10(ROC3)*3+SMA10(ROC4)*4 }
\end{aligned}
$$

\subsection{Gated Recurrent Unit (GRU)}

Long short-term memory (LSTM) and GRU networks are recurrent neural networks (RNN) that do not suffer from vanishing/exploding gradient descent problem associated with simple RNN's. GRU is similar to LSTM network but its structure is simple [12]. It uses only two gates and fewer parameters than LSTM. Therefore, GRU networks can be trained faster than LSTM networks. Besides, GRU networks can generalize with fewer data. LSTM networks have higher expressive power than GRU networks and therefore results in better performance when working with vast amounts of data. However, when working with a moderate volume of data, the GRU network gives better performance than the LSTM network [32]. The mathematical formulation of the GRU network is given in Equation 4 and GRU architecture is given in Figure 1.

$$
\begin{aligned}
& \mathrm{Z}_{\mathrm{t}}=\sigma\left(\mathrm{W}_{\mathrm{z}} \mathrm{x}_{\mathrm{t}}+\mathrm{U}_{\mathrm{z}} \mathrm{H}_{\mathrm{t}-1}\right) \\
& \mathrm{R}_{\mathrm{t}}=\sigma\left(\mathrm{W}_{\mathrm{r}} \mathrm{x}_{\mathrm{t}}+\mathrm{U}_{\mathrm{r}} \mathrm{H}_{\mathrm{t}-1}\right) \\
& \mathrm{H}_{\mathrm{t}}^{\prime}=\tanh \left(\mathrm{W}_{\mathrm{h}} \mathrm{x}_{\mathrm{t}}+\left(\mathrm{R}_{\mathrm{t}} \times \mathrm{H}_{\mathrm{t}-1}\right) \mathrm{U}_{\mathrm{h}}\right) \\
& \mathrm{H}_{\mathrm{t}}=\left(\mathrm{Z}_{\mathrm{t}} \times \mathrm{H}_{\mathrm{t}}^{\prime}\right)+\left(\left(1-\mathrm{Z}_{\mathrm{t}}\right) \times \mathrm{H}_{\mathrm{t}-1}\right)
\end{aligned}
$$

where, $\mathrm{Z}$ and $\mathrm{R}$ are update and reset gates and

$\mathrm{H}_{\mathrm{t}}^{\prime}$ and $\mathrm{H}_{\mathrm{t}}$ are candiadte hidden state and hidden state

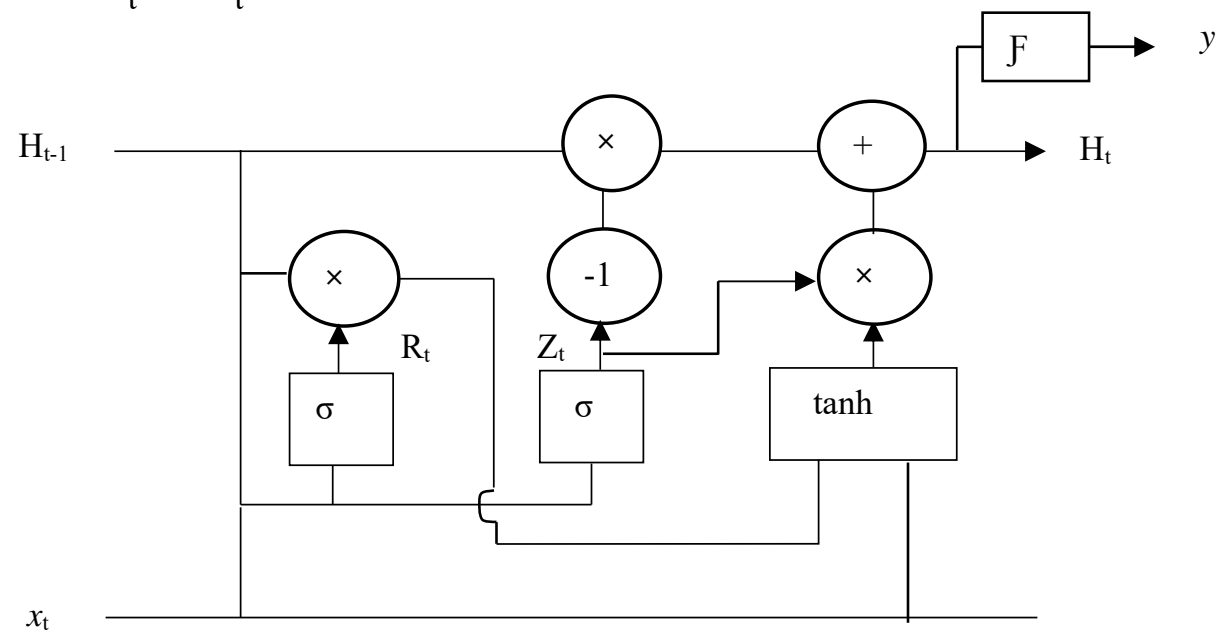

Fig. 1. Architecture of GRU 


\section{Materials and Methods}

This section describes the proposed model, stock data, data pre-processing, data preparation, and configuration of GRU networks used in this research work.

\subsection{Proposed Model}

This research paper proposed a 3-way gated recurrent unit (3-GRU) network architecture to predict the next day's close price. This architecture is a combination of three GRU networks. Component GRU networks of the 3-GRU model predict next day's close price using MACD, KST, and ADX technical indicators. The proposed architecture was inspired by the practices used by technical analysts for stock market forecasting. Technical analysts use each set of technical indicators separately to forecast stock trends and finally combine ideas from these forecasts to make the final prediction. They do not mix-up different technical indicators in an analysis to predict stock price trends. Architecture of the proposed 3-GRU model is shown in Figure 2.

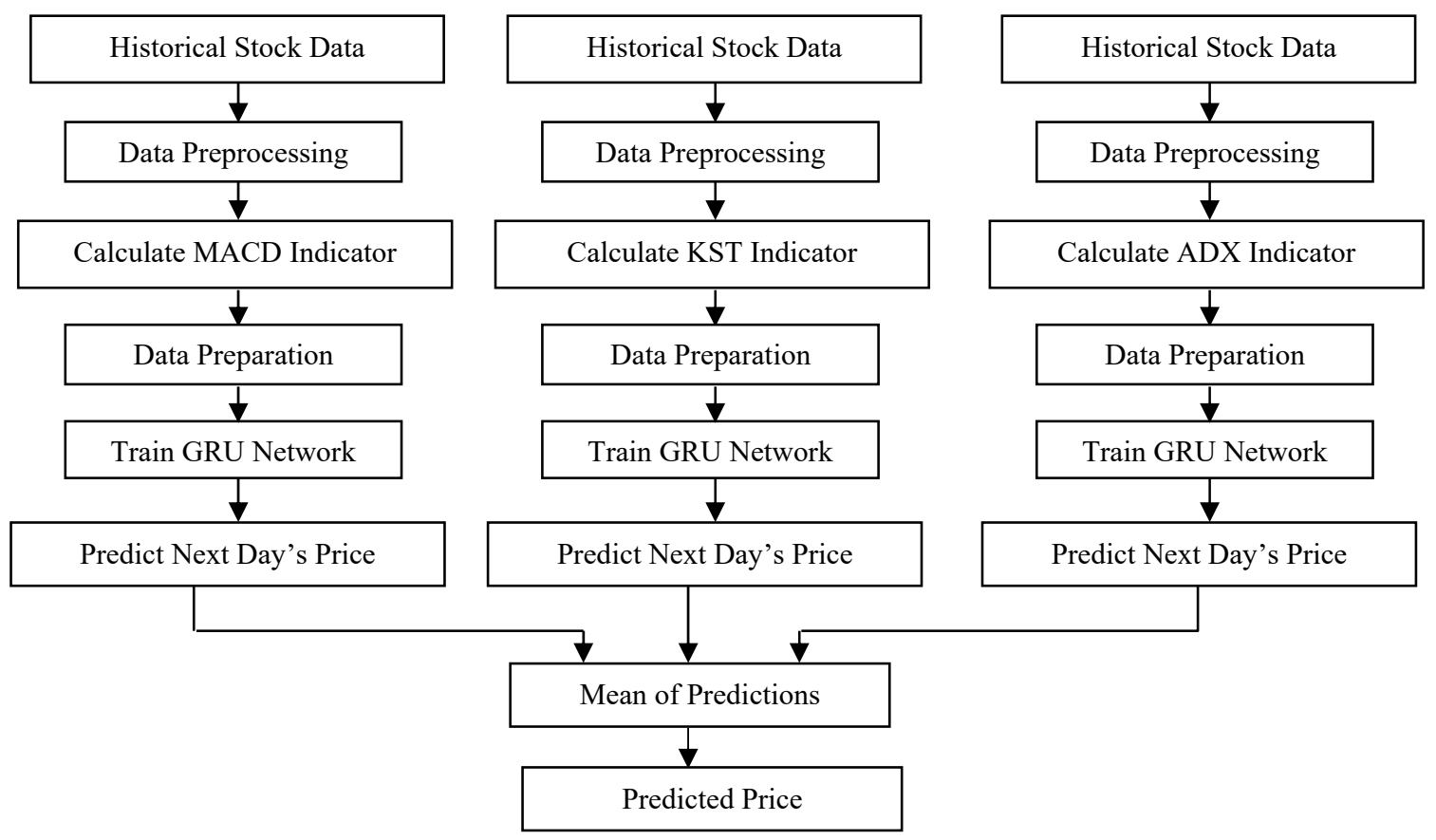

Fig. 2. Architecture of the Proposed 3-GRU Model

\subsection{Stock Trading Data}

The input data used in this research work was historical trading data from the Nepal Stock Exchange (NEPSE), the Bombay Stock Exchange (BSE), the Shanghai Stock Exchange (SSE), and the New York Stock Exchange (NYSE). Three companies were selected randomly from each of the above stock exchanges. The data of BSE stocks was downloaded from BSE India [33]. The downloaded data was daily data from 1st January 2000 to 12th June 2020. The three BSE stocks experimented in this research work were Asian Paints, Kotak Bank, and Shirpur Gold Refinery. The data of NEPSE stocks was collected from Nepal Stock Exchange [34]. The data was daily data from 15th April 2010 to 12th June 2020. The three NEPSE stocks experimented in this research work were Nepal Investment bank (NIB), Standard Chartered Bank (SCB), and Chilime Hydropower (CHCL). Likewise, the data of SSE and NYSE stocks was downloaded from Yahoo Finance [35]. The data of SSE and NYSE stock was daily data from 1st January 2000 to 12th June 2020. The three SSE stocks experimented in this research work were Datang Telecom Technology (DTT), Jiangsu Hengrui Medicine (JHM), and China Merchants Bank (CMB). And the three NYSE stocks experimented in this research work were Marathon Oil (MRO), Boeing Company (BA), and Bank of New York Mellon Corporation (BK).

\subsection{Data Preprocessing}

The downloaded data was first arranged in the order of oldest to newest date and technical indicators MACD, ADX, and KST were calculated. Then, all stock data except the technical indicators and the close price was dropped from the dataset and the target attribute "Next day's Close Price" was generated. The target feature was just close price shifted back by one position. Finally, the input features were normalized using standard scalar.

\subsection{Data Preparation}

The train/validation/test split was done at 8:1:1 ratio. The aim of this study was to predict the close price for day $\mathrm{t}+1$ using input features from $(\mathrm{t}-\mathrm{N}+1)^{\text {th }}$ to $\mathrm{t}^{\text {th }}$ day, where $\mathrm{t}$ is the current trading day. Therefore the input data used in this research work was a combination of $\mathrm{N}$ independent variables $\mathrm{d}_{\mathrm{t}-\mathrm{N}+1} \ldots . \mathrm{d}_{\mathrm{t}-1}, \mathrm{~d}_{\mathrm{t}}$ and a dependent 
variable $c_{t+1}$, where $d_{i}$ is a tuple representing close price and technical indicators and $c_{t+1}$ denotes close price of the day $\mathrm{t}+1$. Here, $\mathrm{d}_{\mathrm{i}}=\left(\mathrm{c}_{\mathrm{i}}, \mathrm{m}_{\mathrm{i}}, \mathrm{ms}_{\mathrm{i}}, \mathrm{ms}_{\mathrm{i}}\right)$ for the GRU-MACD network, $\mathrm{d}_{\mathrm{i}}=\left(\mathrm{c}_{\mathrm{i}}, \mathrm{p}_{\mathrm{i}}, \mathrm{n}_{\mathrm{i}}, \mathrm{a}_{\mathrm{i}}\right)$ for the GRU-ADX network, and $\mathrm{d}_{\mathrm{i}}=\left(\mathrm{c}_{\mathrm{i}}, \mathrm{k}_{\mathrm{i}}, \mathrm{ks}_{\mathrm{i}}, \mathrm{kh}_{\mathrm{i}}\right)$ for the GRU-KST network, where $\mathrm{m}_{\mathrm{i}}$ is MACD, ms is MACD signal, $\mathrm{mh}_{\mathrm{i}}$ is MACD histogram, $\mathrm{p}_{\mathrm{i}}$ is +DMI, $\mathrm{n}_{\mathrm{i}}$ is -DMI, $\mathrm{a}_{\mathrm{i}}$ is ADX, $\mathrm{k}_{\mathrm{i}}$ is KST, $\mathrm{ks}_{\mathrm{i}}$ is KST signal, and $\mathrm{kh}_{\mathrm{i}}$ is $\mathrm{KST}$ histogram.

\subsection{Model Configuration}

Configuration of all component GRU networks used in this research work was $4 \times 128 \times 128 \times 1$ and the configuration of GRU-ALL network was $10 \times 256 \times 256 \times 1$. Each hidden layer of the networks was followed by a dropout layer. Hyperbolic tangent activation function was used in the hidden layers and linear activation function was used in the output layer of all GRU network. In addition, Adam optimizer was used in all GRU networks. The dimension of input to all component GRU networks was $(\mathrm{L}, \mathrm{N}, 4)$ and the dimension of input to the GRU-ALL model was ( $\mathrm{L}, \mathrm{N}, 10)$, where $\mathrm{L}$ is the length of the dataset, $\mathrm{N}$ is the window size and the last number is the number of input features. Window size 5 was used in all models as suggested by Saud and Shakya [36].

\section{Results and Discussion}

In this research work, ten experiments were conducted to determine the mean squared error (MSE) for all GRU network. Further, the standard deviation of mean squared errors obtained from each model was calculated. This section presents comparison among these matrices.

Table 1. Experimental result of NEPSE stocks

\begin{tabular}{|l|l|l|l|l|l|l|}
\hline & Stock Symbol & GRU-MACD & GRU-KST & GRU-ADX & 3-GRU & GRU-ALL \\
\hline \multirow{4}{*}{$\begin{array}{l}\text { Average } \\
\text { MSE }\end{array}$} & NIB & 64.10 & 66.04 & 73.94 & $\mathbf{6 1 . 1 4}$ & 74.14 \\
\cline { 2 - 7 } & SCB & 267.34 & 262.49 & 274.09 & $\mathbf{2 5 6 . 9 5}$ & 264.17 \\
\cline { 2 - 7 } & CHCL & 142.84 & 144.42 & 147.11 & $\mathbf{1 3 2 . 0 5}$ & 147.87 \\
\hline \multirow{3}{*}{$\begin{array}{l}\text { Standard } \\
\text { Deviation }\end{array}$} & NIB & 5.32 & 4.53 & 6.51 & $\mathbf{3 . 7 0}$ & 7.2 \\
\cline { 2 - 7 } & SCB & 10.18 & 18.05 & 19.65 & $\mathbf{7 . 7 4}$ & 8.55 \\
\cline { 2 - 7 } & CHCL & 18.72 & 14.32 & 4.43 & $\mathbf{3 . 1 1}$ & 20.05 \\
\hline
\end{tabular}

Table 2. Experimental result of BSE stocks

\begin{tabular}{|l|l|l|l|l|l|l|}
\hline & Stock Symbol & GRU-MACD & GRU-KST & GRU-ADX & 3-GRU & GRU-ALL \\
\hline \multirow{3}{*}{$\begin{array}{l}\text { Average } \\
\text { MSE }\end{array}$} & Asian & 1147.20 & $\mathbf{1 0 6 1 . 1 5}$ & 1156.21 & 1074.30 & 1122.08 \\
\cline { 2 - 7 } & Kotak & 471.21 & 457.09 & 466.30 & $\mathbf{4 3 4 . 7 6}$ & 464.28 \\
\cline { 2 - 7 } & Shirpur & 5.16 & 3.90 & 4.97 & $\mathbf{3 . 2 4}$ & 6.27 \\
\hline \multirow{3}{*}{$\begin{array}{l}\text { Standard } \\
\text { Deviation }\end{array}$} & Asian & 91.16 & 38.73 & 101.32 & $\mathbf{3 8 . 2 3}$ & 98.02 \\
\cline { 2 - 7 } & Kotak & 39.12 & 30.52 & 20.47 & $\mathbf{1 0 . 0 4}$ & 63.43 \\
\cline { 2 - 7 } & Shirpur & 1.38 & 0.87 & 1.25 & $\mathbf{0 . 4 0}$ & 0.96 \\
\hline
\end{tabular}

Table 3. Experimental result of SSE stocks

\begin{tabular}{|l|l|l|l|l|l|l|}
\hline & Stock Symbol & GRU-MACD & GRU-KST & GRU-ADX & 3-GRU & GRU-ALL \\
\hline \multirow{4}{*}{$\begin{array}{l}\text { Average } \\
\text { MSE }\end{array}$} & DTT & 0.191 & 0.182 & 0.188 & $\mathbf{0 . 1 7 6}$ & 0.196 \\
\cline { 2 - 7 } & JHM & 4.37 & 4.35 & 4.41 & $\mathbf{4 . 0 8}$ & 4.24 \\
\cline { 2 - 7 } & CMB & $\mathbf{0 . 3 8 3}$ & 0.407 & 0.423 & 0.387 & 0.391 \\
\hline \multirow{3}{*}{$\begin{array}{l}\text { Standard } \\
\text { Deviation }\end{array}$} & DTT & 0.016 & 0.011 & 0.011 & $\mathbf{0 . 0 0 3}$ & 0.015 \\
\cline { 2 - 7 } & JHM & 0.28 & 0.53 & 0.22 & $\mathbf{0 . 0 9}$ & 0.28 \\
\cline { 2 - 7 } & CMB & 0.014 & 0.020 & 0.015 & $\mathbf{0 . 0 0 4}$ & 0.015 \\
\hline
\end{tabular}


Table 4. Experimental result of NYSE stocks

\begin{tabular}{|l|l|l|l|l|l|l|}
\hline & Stock Symbol & GRU-MACD & GRU-KST & GRU-ADX & 3-GRU & GRU-ALL \\
\hline \multirow{4}{*}{$\begin{array}{l}\text { Average } \\
\text { MSE }\end{array}$} & MRO & 0.257 & 0.247 & 0.273 & $\mathbf{0 . 2 4 3}$ & 0.290 \\
\cline { 2 - 7 } & BA & 103.42 & 109.86 & 112.18 & $\mathbf{1 0 2 . 0 7}$ & 111.04 \\
\cline { 2 - 7 } & BK & 0.946 & 0.906 & 0.941 & $\mathbf{0 . 9 0 0}$ & 1.018 \\
\hline \multirow{3}{*}{$\begin{array}{l}\text { Standard } \\
\text { Deviation }\end{array}$} & MRO & 0.033 & 0.017 & 0.044 & $\mathbf{0 . 0 1 6}$ & 0.032 \\
\cline { 2 - 7 } & BA & $\mathbf{5 . 6 1}$ & 19.06 & 17.78 & 7.59 & 10.53 \\
\cline { 2 - 7 } & BK & 0.043 & 0.035 & 0.037 & $\mathbf{0 . 0 2 0}$ & 0.087 \\
\hline
\end{tabular}

The experimental results obtained from the NEPSE, BSE, SSE, and NYSE stocks are presented in Table 1 to Table 4 respectively. From the results, we observed that 3-GRU model predicted the next day's close price with the lowest average MSE for the stocks NIB, SCB, CHCL, Kotak, Shirpur, DTT, JHM, MRO, BA, and BK. The model achieved the specified prediction with the lowest standard deviation for the stocks NIB, SCB, CHCL, Asian, Kotak, Shirpur, DTT, JHM, CMB, MRO, and BK. Besides, the GRU-KST and GRU-MACD models are able to predict next day's close price with the lowest MSE for the stocks Asian and CMB respectively. And the GRU-MACD model achieved the lowest standard deviation for the stock BA.

On the other side, GRU-ALL model predicted the next day's close price with the highest average MSE for the stocks NIB, CHCL, Shirpur, DTT, MRO, and BK. GRU-ADX model predicted the next day's close price with the highest average MSE for the stocks SCB, Asian, Kotak, JHM, CMB, and BA. Further, the GRU-ALL model predicted the specified next day's close price with the highest standard deviation for the stocks NIB, CHCL, Kotak, and BK. The GRU-ADX model predicted the specified next day's close price with the highest standard deviation for the stocks SCB, Asian, and MRO. The GRU-MACD model achieved the highest standard deviation for the stocks Shirpur and DTT. And the GRU-KST model achieved the highest standard deviation for the stocks JHM, CMB, and BA.

In summary, 3-GRU model was able to predict next day's close price with the lowest average MSE and lowest standard deviation for most of the stocks from all stock exchanges considered in the research work. At the same time, GRU-ALL model predicted the next day's close price with the highest average MSE and standard deviation for majority stocks. In the case of component GRU networks, GRU-ADX model performed worse than GRU-KST and GRU-MACD model whereas GRU-KST model performed better.

\section{Conclusion}

Many researchers from the field of computer science, statistics, and finance are involving in stock price forecasting research. Technical indicators are widely used by the researchers for predicting stock prices. This research paper proposed a 3-GRU model to predict the next day's close price and compared its performance with GRU-MACD, GRU-KST, GRU-ADX, and GRU-ALL models. All models were evaluated in terms of average MSE and standard deviation of MSE's achieved from predicting the next day's close price.

From the experimental results of 12 stocks, we observed that 3-GRU model is able to predict next day's close price with the lowest average MSE for 10 stocks. The model achieved this prediction with the lowest standard deviation for 11 stocks. On the other hand, both GRU-ALL and GRU-ADX models achieved the highest average MSE for 6 stocks. In addition, GRU-ALL, GRU-ADX, GRU-MACD, and GRU-KST model predicted the next day's stock price with the highest standard deviation for four, three, two, and three stocks respectively. From this observation we concluded following three facts: (1) GRU-ADX is inefficient than GRUMACD and GRU-KST models and hence we concluded that ADX indicator is least suitable among other indicators for predicting next day's close price (2) GRU-ALL model is most inefficient among all 5 models and hence we concluded that combining different technical indicators into one feature vector and using it as input to stock price prediction model is not a good idea (3) 3-GRU model is the most efficient model among all other models and hence we concluded that it is the better model for predicting next day's close price.

\section{References}

[1] Hegazy, O.; Soliman, O. S.; and Salam, M. A. (2013): A Machine Learning Model for Stock Market Prediction. Int. J. Comput. Sci. Telecommun., 4(12), pp. 17-23.

[2] Dhole, M. (2017): Review paper on Fundamental and Technical Analysis. Elixir Financ. Manag., 103(2017), pp. 45524-45525.

[3] Kirkpatrick, C. D and Julie D. A., (2010). Technical Analysis: The Complete Resource for Financial Market Technicians. PHI Publication, Second Edition, Delhi.

[4] Lo, A. W. and Hasanhodzic J. (2010). The Evolution of Technical Analysis: Financial Prediction from Babylonian Tablets to Bloomberg Terminals. Bloomberg Press, First Edition, New York.

[5] Abu-Mostafa, Y. S. and Atiya A. F. (1996): Introduction to financial forecasting. Appl. Intell., 6, pp. 205-213. 
[6] Lin, Y.; Guo, H. and Hu, J. (2013): An SVM-based approach for stock market trend prediction. In Proceedings of International Joint Conference on Neural Networks (IJCNN), Dallas, USA, pp. 1-7.

[7] Booth, A.; Gerding, E. and McGroarty, F. (2014): Predicting equity market price impact with performance weighted ensembles of random forests, In Proceedings of IEEE Conference on Computational Intelligence for Financial Engineering \& Economics (CIFEr), London, UK, pp. 286-293s.

[8] Guresen, E.; Kayakutlu, G. and Daim, T. U. (2011): Using artificial neural network models in stock market index prediction. Expert Syst. Appl., 38(8), pp. 10389-10397.

[9] Lipton, Z. C.; Berkowitz, J. and Elkan, C. (2015): A Critical Review of Recurrent Neural Networks for Sequence Learning, arXiv: 1506.00019

[10] Stoean, C.; Paja, W.; Stoean, R.; and Sandita, A. (2019): Deep architectures for long-term stock price prediction with a heuristic-based strategy for trading simulations. PLoS One, 14(10), Id:e0223593.

[11] Lien, M. D.; Sadeghi-Niaraki, A.; Huy, H. D.; Min, K. and Moon, H. (2018): Deep learning approach for short-term stock trends prediction based on two-stream gated recurrent unit network. IEEE Access, 6, pp. 55392 - 55404.

[12] Cho, K. (2014): Learning phrase representations using RNN encoder-decoder for statistical machine translation. In Proceedings of Conference on Empirical Methods in Natural Language Processing, Doha, Qatar, pp. 1724-1734.

[13] Hochreiter, S and Schmidhuber, J. (1997): Long Short-Term Memory. Neural Comput., 9(8), pp. 1735-1780.

[14] Jabbarzadeh, A.; Shavvalpour, S.; Khanjarpanah, H. and Dourvash, D. (2016): A multiple-criteria approach for forecasting stock price direction: Nonlinear probability models with application in S\&P 500 index. Int. J. Appl. Eng. Res., 11(6), pp. 3870-3878.

[15] Chen, Y. and Hao, Y. (2017): A feature weighted support vector machine and K-nearest neighbor algorithm for stock market indices prediction. Expert Syst. Appl., 80, pp. 340-355.

[16] Rodríguez-González, A.; García-Crespo, A.; Colomo-Palacios, R.; Guldrís Iglesias, F. and Gómez-Berbís, J. M. (2011): CAST: Using neural networks to improve trading systems based on technical analysis by means of the RSI financial indicator. Expert Syst. Appl., 38(9), pp. 11489-11500.

[17] Chen, Y. S.; Cheng, C. H. and Tsai, W. L. (2014): Modeling fitting-function-based fuzzy time series patterns for evolving stock index forecasting. Appl. Intell. 41, pp. 327-347.

[18] Patel, J.; Shah, S.; Thakkar, P. and Kotecha, K. (2015): Predicting stock and stock price index movement using Trend Deterministic Data Preparation and machine learning techniques. Expert Syst. Appl, 42, pp. 259-268.

[19] Chiang, W. C.; Enke, D.; Wu, T. and Wang, R. (2016): An adaptive stock index trading decision support system. Expert Syst. Appl., 59, pp. 195-207.

[20] Shynkevich, Y.; McGinnity, T. M.; Coleman, S. A.; Belatreche, A. and Li, Y. (2017): Forecasting price movements using technical indicators: Investigating the impact of varying input window length. Neurocomputing., 264, pp. 71-88.

[21] Long, W.; Lu, Z. and Cui, L. (2019): Deep learning-based feature engineering for stock price movement prediction. KnowledgeBased Syst.,164, pp. 163-173.

[22] Kim, T. and Kim, H. Y. (2019): Forecasting stock prices with a feature fusion LSTM-CNN model using different representations of the same data. PLoS One., 14(2). Id:e0212320.

[23] Gao, T. and Chai, Y. (2018): Improving Stock Closing Price Prediction Using Recurrent Neural Network and Technical Indicators. Neural Comput., 30(7), pp. 1-22.

[24] Atsalakis, G. S. and Valavanis, K. P. (2009): Surveying stock market forecasting techniques - Part II: Soft computing methods. Expert Syst. Appl., 36(3), pp. 5932-5941.

[25] Olson, D. and Mossman, C. (2003): Neural network forecasts of Canadian stock returns using accounting ratios. Int. J. Forecast., 19(3), pp. 453-465.

[26] Technical Analysis Library in Python: Documentation, Accessed March 2020. Available at: https://technical-analysis-library-inpython.readthedocs.io/en/latest/.

[27] M. J. Pring (2014): Technical Analysis Explained: The Successful Investor's Guide to Spotting Investment Trends and Turning Points. Fifth Edition, McGraw Hill, New York

[28] Wilder, J. W. (1978): New Concepts in Technical Trading Systems Hardcover. First Edition, Trend Research Publishing.

[29] Appel, G. (2005): Technical Analysis: Power Tools for Active Investors, First Edition. Financial Times Press, New Jersey.

[30] Nguyen, T. T. and Yoon, S. (2019): Novel Approach to Short-Term Stock Price Movement Prediction using Transfer Learning. Appl. Sci.., $9(22)$, pp. 4745

[31] Colby, R.W. (2002): The Encyclopedia Of Technical Market Indicators. Second Edition, McGraw-Hill, New York.

[32] Weiss, G.; Goldberg, Y. and Yahav, E. (2018): On the practical computational power of finite precision RNNs for language recognition. In 56th Annual Meeting of the Association for Computational Linguistics, Proceedings of the Conference, Melbourne, Australia, pp. 740-745.

[33] S\&P BSE SENSEX Stock Prices, Accessed March 2020. Available at:https://www.bseindia.com/markets/equity/EQReports/ StockPrcHistori.aspx.

[34] Nepal Stock Exchange Ltd, Accessed March 2020. Available at: http://nepalstock.com.np/stockWisePrices.

[35] Yahoo Finance, Accessed March 2020. Available at: https://finance.yahoo.com/.

[36] Saud, A. S. and Shakya, S. (2020): Analysis of look back period for stock price prediction with RNN variants: A case study on banking sector of NEPSE. Procedia Comput. Sci., 167, pp. 788-798. 